\title{
Stability of trions in coupled quantum wells modelled by two-dimensional bilayers
}

\author{
O. Witham,,$^{1,2}$ R. J. Hunt ${ }^{2}$ and N. D. Drummond ${ }^{2}$ \\ ${ }^{1}$ Institut für Theoretische Physik, Goethe-Universität Frankfurt, 60438 Frankfurt am Main, Germany \\ ${ }^{2}$ Department of Physics, Lancaster University, Lancaster LA1 4YB, United Kingdom
}

(Dated: October 16, 2017)

\begin{abstract}
We report variational and diffusion quantum Monte Carlo calculations of the binding energies of indirect trions and biexcitons in ideal two-dimensional bilayer systems within the effective-mass approximation, and with a Coulomb $1 / r$ interaction between charge carriers. The critical layer separation at which trions become unbound has been studied for various electron-hole mass ratios, and found to be over an order of magnitude larger than the critical layer separation for biexcitons.
\end{abstract}

PACS numbers: 71.35.Cc, 71.35.Pq, 78.67.De, 02.70.Ss

\section{INTRODUCTION}

Excitonic complexes consisting of bound states of small numbers of electrons and holes have been observed under many different conditions in semiconductors. In the dilute limit, excitons collectively behave as a gas of weakly interacting neutral bosons. It was first predicted in the 1960s that Bose-Einstein condensates of excitons might form under experimentally accessible conditions, $\frac{112}{, 2}$ and coupled quantum-well heterostructures, in which electrons and holes are confined to spatially separated layers, were later identified as an ideal testbed for the observation of an excitonic condensate ${ }^{3 / 4}$ Such geometries hinder recombination and significantly increase the exciton lifetime, which is necessary if thermalization of a photoexcited gas of excitons is to occur. Furthermore, at sufficiently large layer separations, the repulsive dipole-dipole interaction between spatially indirect excitons helps to prevent the formation of larger chargecarrier complexes.

A Bose-Einstein condensate of excitons in a semiconductor such as GaAs is expected to be dark, i.e., not to couple directly to photons. ${ }^{5}$ Striking fragmented-ring patterns of indirect exciton photoluminescence have been observed around laser excitation spots in GaAs coupled quantum wells, $\frac{617}{6}$ and subsequent work ${ }^{[8}$ has indicated that these patterns arise due to indirect excitons travelling outwards in a dark, coherent state from the point at which they are created. More recent experimental work has provided further evidence for the creation of an excitonic Bose-Einstein condensate in coupled quantum wells, as revealed by the macroscopic spatial coherence of indirect excitons at low temperatures. ${ }^{\frac{9}{12}}$ However, despite the many exciting and important advances in this field, the situation is not perfectly clearcut and there remains a need to analyze factors that could influence the formation of an excitonic condensate in a coupled quantum well. 13

The formation of indirect biexcitons (also neutral composite bosons, but with a higher mass than excitons) could potentially inhibit condensation, but various theoretical works $s^{14} 17$ showed that biexcitons are expected to be unbound in the typical coupled quantum-well geome- tries accessible to experimentalists, in which the layer separation is of the order of tens of Angströms. In this work we investigate the stability of a different class of charge-carrier complex, namely indirect trions (bound states of two electrons in one layer and a hole in the opposite layer, or vice versa) in coupled quantum wells. Trion formation can occur if a nonzero concentration of free charge carriers is present in a semiconductor, or if the different mobilities of electrons and holes leads to a local imbalance in the carrier concentration. Free charge carriers can then bind to excitons created by optical excitation, producing trions. A finite concentration of trions effectively provides disorder, and could therefore tend to restrict Bose-Einstein condensation of the remaining excitons. As an additional motivation for our work, it is known that trions play a key role in the optical properties of atomically thin transition-metal dichalcogenides and other two-dimensional (2D) semiconductors ${ }^{[1823} \mathrm{We}$ shall assume an isotropically screened Coulomb interaction, as appropriate for trions in GaAs/AlGaAs heterostructures, rather than the Keldysh interaction appropriate for charge carriers in 2D systems with a significant in-plane polarizability. ${ }^{24 \mid 25}$ Nevertheless, our results are of qualitative relevance to optical studies of bilayers of $2 \mathrm{D}$ semiconductors.

We have used the variational and diffusion quantum Monte Carlo (VMC and DMC) methods ${ }^{26 \mid 27}$ to calculate the ground-state energies and binding energies of spatially indirect trions within the 2D-isotropic effectivemass approximation. A negative trion in such an ideal 2D bilayer system is approximately described by the Hamiltonian

$$
\begin{gathered}
\hat{H}=-\frac{\hbar^{2}}{2 m_{\mathrm{e}}} \nabla_{\mathrm{e}_{1}}^{2}-\frac{\hbar^{2}}{2 m_{\mathrm{e}}} \nabla_{\mathrm{e}_{2}}^{2}-\frac{\hbar^{2}}{2 m_{\mathrm{h}}} \nabla_{\mathrm{h}}^{2}+\frac{e^{2}}{4 \pi \epsilon r_{\mathrm{ee}}} \\
-\frac{e^{2}}{4 \pi \epsilon \sqrt{r_{\mathrm{e}_{1} \mathrm{~h}}^{2}+d^{2}}}-\frac{e^{2}}{4 \pi \epsilon \sqrt{r_{\mathrm{e}_{2} \mathrm{~h}}^{2}+d^{2}}}
\end{gathered}
$$

where $m_{\mathrm{e}}$ and $m_{\mathrm{h}}$ are the electron and hole effective masses, $e$ is the magnitude of electronic charge, $d$ is the interlayer separation, and $\epsilon$ is the permittivity of the medium in which the two layers are embedded. The inplane interparticle distances are given by $r_{\mathrm{ee}}=\left|\mathbf{r}_{\mathrm{e}_{1}}-\mathbf{r}_{\mathrm{e}_{2}}\right|$ and $r_{\mathrm{e}_{i} \mathrm{~h}}=\left|\mathbf{r}_{\mathrm{e}_{i}}-\mathbf{r}_{\mathrm{h}}\right|$, where $\mathbf{r}_{\mathrm{e}_{1}}, \mathbf{r}_{\mathrm{e}_{2}}$, and $\mathbf{r}_{\mathrm{h}}$ are $2 \mathrm{D}$ vec- 
tors holding the in-plane coordinates of the electrons and the hole, which are assumed to be confined to parallel planes as shown in Fig. 1. Although our results pertain directly to the negative trion $\left(\mathrm{X}^{-}\right)$, the corresponding properties of the positive trion $\left(\mathrm{X}^{+}\right)$can easily be generated by charge conjugation, i.e., by interchanging $m_{\mathrm{e}}$ and $m_{\mathrm{h}}$ (or, equivalently, by replacing $\sigma$ by $\sigma^{-1}$ ). Furthermore, we consider only the ground-state case in which the two electrons are distinguishable (opposite-spin electrons). Trions and biexcitons with indistinguishable electrons are much less stable than trions with distinguishable electrons; by analogy with results obtained for biexcitons in single-layer 2D semiconductors, we expect that trions and biexcitons with indistinguishable particles are only stable when the indistinguishable particles are very heavy, so that exchange effects are negligible.25

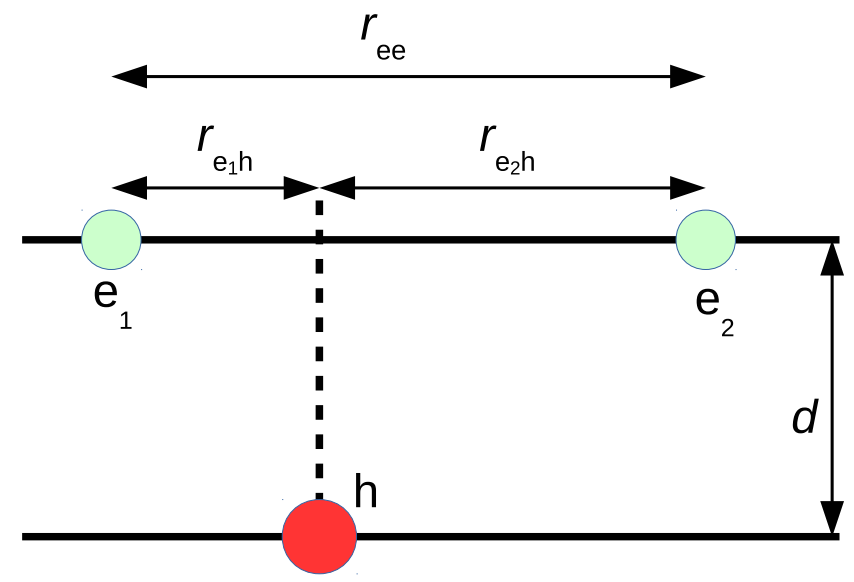

FIG. 1. (Color online) Schematic arrangement of two electrons (light green) and a hole (red) for a negative trion in a coupled quantum well, showing the definition of the interparticle distances. The two electrons are in a spin singlet state and hence act as distinguishable particles in the spatial wave function. The electrons and hole move in 2D in spatially separated layers.

Although biexcitons in coupled quantum wells have been studied extensively, there are relatively few theoretical studies of indirect trions. Kulakovskii and Lozovik ${ }^{28}$ and Berman et al ${ }^{29}$ study trions consisting of a direct exciton in one layer bound to a charge carrier in a neighboring layer, and make the interesting suggestion that Wigner crystallization of trions could occur. Kovalev and Chaplik ${ }^{30}$ investigated the behavior of indirect trions in an out-of-plane magnetic field by treating the interlayer electron-hole Coulomb potential within a harmonic approximation. Sergeev and Suris ${ }^{31}$ studied a model similar to ours within a heavy-hole approximation. We compare our results with theirs in Sec. IIIA.

The rest of this paper proceeds as follows. In Sec. II we describe our methodology for calculating the binding energies of trions and biexcitons; in Sec. III we present our results; and finally in Sec. IV we draw our conclu- sions. Except where otherwise stated, we use excitonic units: energies are given in terms of the exciton Rydberg $R_{\mathrm{y}}^{*}=\mu e^{4} /\left[2(4 \pi \epsilon \hbar)^{2}\right]$ and lengths are given in terms of the exciton Bohr radius $a_{0}^{*}=4 \pi \epsilon \hbar^{2} /\left(\mu e^{2}\right)$, where $\mu=m_{\mathrm{e}} m_{\mathrm{h}} /\left(m_{\mathrm{e}}+m_{\mathrm{h}}\right)$ is the reduced mass of the electron and hole. In these units the dimensionless solutions $E / R_{\mathrm{y}}^{*}$ to the Schrödinger equation only depend on the electron-hole mass ratio $\sigma=m_{\mathrm{e}} / m_{\mathrm{h}}$ and the dimensionless layer separation $d / a_{0}^{*}$.

\section{COMPUTATIONAL METHODOLOGY}

The VMC and DMC methods ${ }^{26127}$ as implemented in the CASINO code ${ }^{32}$ were used in conjunction with a trial wave function of the form

$$
\Psi=\exp (J) \Psi_{\mathrm{ee}} \Psi_{\mathrm{e}_{1} \mathrm{~h}} \Psi_{\mathrm{e}_{2} \mathrm{~h}},
$$

where

$$
\begin{aligned}
\Psi_{\mathrm{ee}} & =\exp \left[\frac{c_{1} r_{\mathrm{ee}}}{1+c_{2} r_{\mathrm{ee}}}+\frac{1}{2}\left(e^{-c_{6} r_{\mathrm{ee}}^{2}}-1\right) \log \left(r_{\mathrm{ee}}\right)\right] \\
\Psi_{\mathrm{e}_{1} \mathrm{~h}} & =\exp \left[\frac{c_{3} r_{\mathrm{e}_{1} \mathrm{~h}}+c_{4} r_{\mathrm{e}_{1} \mathrm{~h}}^{2}}{1+c_{5} r_{\mathrm{e}_{1} \mathrm{~h}}}\right] \\
\Psi_{\mathrm{e}_{2} \mathrm{~h}} & =\exp \left[\frac{c_{3} r_{\mathrm{e}_{2} \mathrm{~h}}+c_{4} r_{\mathrm{e}_{2} \mathrm{~h}}^{2}}{1+c_{5} r_{\mathrm{e}_{2} \mathrm{~h}}}\right]
\end{aligned}
$$

to perform total-energy calculations for the negative trion. Our trial wave function is similar to the one used by Tan et al. to study biexcitons, ${ }^{33}$ but with an additional term that describes the behavior of the wave function when one electron is far from the remaining exciton. The nested exponential in Eq. (3) acts as a "switching on" term; the logarithm only manifests appreciably in the case that the two electrons are far apart. The Jastrow exponent $J$ contains cuspless two-body and threebody polynomial functions of the interparticle distances, truncated at finite range $\frac{34 \mid 35}{35}$ The wave function of Eqs. (2)-(5) incorporates both short- and long-range effects through the use of the Padé-form exponents. It exhibits the correct symmetry of the ground-state system, being invariant upon the exchange of the two electrons, i.e., $\Psi\left(\mathbf{r}_{\mathrm{e}_{1}}, \mathbf{r}_{\mathrm{e}_{2}}, \mathbf{r}_{\mathrm{h}}\right)=\Psi\left(\mathbf{r}_{\mathrm{e}_{2}}, \mathbf{r}_{\mathrm{e}_{1}}, \mathbf{r}_{\mathrm{h}}\right)$. The trial wave function reduces to

$$
\Psi \rightarrow A \frac{1}{\sqrt{r_{\mathrm{ee}}}} \exp \left[-k r_{\mathrm{ee}}\right] \exp \left[\frac{c_{3} r_{\mathrm{e}_{2} \mathrm{~h}}+c_{4} r_{\mathrm{e}_{2} \mathrm{~h}}^{2}}{1+c_{5} r_{\mathrm{e}_{2} \mathrm{~h}}}\right],
$$

when one electron is far from the remaining exciton, where $k>0$ and $A$ is constant. This form of wave function is appropriate for an electron moving in the potential energy due to the static charge distribution of the remaining indirect exciton. The static dipole moment of an exciton is $\mathbf{p}=-e d \mathbf{e}_{z}$, where $\mathbf{e}_{z}$ is a unit vector in the $z$ direction. Let the separation of the electron from the 
center of the exciton be $\mathbf{r}+(d / 2) \mathbf{e}_{z}$, where $\mathbf{r}$ is the inplane separation. Hence the long-range exciton-electron interaction energy is

$$
-e \mathbf{p} \cdot\left[\mathbf{r}+(d / 2) \mathbf{e}_{z}\right] /\left[4 \pi \epsilon\left|\mathbf{r}+(d / 2) \mathbf{e}_{z}\right|^{3}\right] \sim r^{-3} .
$$

Solving the 2D radial Schrödinger equation for motion in a rapidly decaying potential such as $r^{-3}$ gives the form of wave function shown in Eq. (6) at long range.

The parameter set $\left\{c_{1}, \ldots, c_{6}\right\}$ is subject to the following conditions: (i) The values of $c_{1}$ and $c_{3}$ are fixed by the electron-electron and electron-hole Kato cusp conditions $\frac{36137}{\text { (ii) }} c_{4}<0$ to ensure that the wave function falls off exponentially as $r_{\mathrm{e}_{i} \mathrm{~h}} \rightarrow \infty$; (iii) $c_{2}, c_{5}>0$ to avoid divergences in the wave function; and (iv) $c_{6}>0$ to enforce the correct long-range behavior of an electron in a dipole field. The optimal values of the parameters $\left\{c_{1}, \ldots, c_{6}\right\}$ were obtained by successive minimization of the variance of the local energy and the energy expectation value. 38$] 40$

In the DMC method the ground-state component of the trial wave function is projected out by simulating a stochastic process governed by the Schrödinger equation in imaginary time. In systems such as those considered here, in which there are no indistinguishable fermions, there are no uncontrolled approximations in the DMC ground-state energy. We simultaneously remove time-step bias and population-control bias by performing DMC calculations at two different, small time steps, with the walker population being in inverse proportion to the time step, and extrapolating linearly to zero time step.

The binding energy $E_{\mathrm{X}^{-}}^{\mathrm{b}}$ of a negative trion is defined as the energy required to split the trion into an exciton and a free electron, i.e.,

$$
E_{\mathrm{X}^{-}}^{\mathrm{b}}=E_{\mathrm{X}}-E_{\mathrm{X}^{-}},
$$

where $E_{\mathrm{X}}$ and $E_{\mathrm{X}^{-}}$are the ground-state total energies of an exciton and a negative trion, respectively. Instability of the trion with respect to dissociation into a free electron and an indirect exciton is signalled by difficulty optimizing a bound-state trial wave function, followed by the occurrence of nonpositive $E_{\mathrm{X}^{-}}^{\mathrm{b}}$ values in DMC calculations in which the trial wave function is forced to be bound. The curve defined by $E_{\mathrm{X}^{-}}^{\mathrm{b}}\left(d / a_{0}^{*}, \sigma\right)=0$, where $E_{\mathrm{X}^{-}}^{\mathrm{b}}\left(d / a_{0}^{*}, \sigma\right)$ is the binding energy of the trion with a given electron-hole mass ratio $\sigma$ and dimensionless layer separation $d / a_{0}^{*}$, defines the boundary of the stability region of the trion. In practice we invert this relation and simply quote the critical layer separation $d_{\mathrm{X}^{-}}^{\text {crit }}(\sigma)$ as a function of mass ratio. We have attempted to probe the trion stability region directly by studying systems with electron-hole mass ratios $\sigma=1 / 4,1 / 2,3 / 4,1,4 / 3,2$, and 4 , and fitting the resultant trion binding energies to a Padé approximant of the form

$$
\frac{E_{\mathrm{X}^{-}}^{\mathrm{b}}\left(d / a_{0}^{*}\right)}{R_{\mathrm{y}}^{*}}=\frac{E_{\mathrm{X}^{-}}^{\mathrm{b}}(0) / R_{\mathrm{y}}^{*}+\sum_{i=1}^{3} a_{i}\left(d / a_{0}^{*}\right)^{i}}{1+\sum_{j=1}^{4} b_{j}\left(d / a_{0}^{*}\right)^{j}},
$$

where $\left\{a_{i}\right\}$ and $\left\{b_{j}\right\}$ are fitting parameters. We have found that this functional form yields sufficiently accurate fits of the binding energies for all mass ratios considered here.

Where the trion is bound, we have performed fits to a "partial 2D" Padé approximant of the form

$$
\frac{E_{\mathrm{X}^{-}}^{\mathrm{b}}\left(\sigma, d / a_{0}^{*}\right)}{R_{\mathrm{y}}^{*}}=\frac{\sum_{i=0}^{3} \sum_{j=0}^{3} f_{i j}(1+\sigma)^{-i / 2}\left(d / a_{0}^{*}\right)^{j}}{1+\sum_{k=1}^{4} g_{k}\left(d / a_{0}^{*}\right)^{k}},
$$

where $f_{i j}$ and $g_{k}$ are fitting parameters, and the $\sigma$ dependence is motivated by the harmonic approximation within the Born-Oppenheimer approximation in the case that $\sigma \rightarrow \infty .25|31| 41$

Finally, we report pair-distribution functions (PDFs), which give information about the structure and spatial extent of charge-carrier complexes. For a negative trion, the electron-electron and electron-hole PDFs are defined via

$$
\begin{aligned}
& g_{\mathrm{X}^{-}}^{\mathrm{ee}}(\mathbf{r})=\left\langle\delta\left(\mathbf{r}-\mathbf{r}_{\mathrm{ee}}\right)\right\rangle \\
& g_{\mathrm{X}^{-}}^{\mathrm{eh}}(\mathbf{r})=\left\langle\sum_{i=1}^{2} \delta\left(\mathbf{r}-\mathbf{r}_{\mathrm{e}_{i} \mathrm{~h}}\right)\right\rangle .
\end{aligned}
$$

The PDFs in a biexciton are defined in an analogous fashion. The PDFs are evaluated by binning the interparticle distances sampled in the VMC and DMC calculations. The error in the VMC and DMC estimates of the PDF is first order in the error in the trial wave function; however, the error in the extrapolated estimate of the PDF, given by two times the DMC result minus the VMC result, is second order in the error in the trial wave function. ${ }^{42}$ The PDF results that we report were obtained by extrapolated estimation.

\section{RESULTS AND DISCUSSION}

\section{A. Trion binding energy and region of stability}

Our negative-trion binding-energy results are displayed in Fig. 2, and our predicted critical layer separations are shown in Table I. For larger electron-hole mass ratios $\sigma$, the binding energy decays slowly to zero, and furthermore the DMC calculations become more difficult due to the separation of imaginary-time scales for the different particles. The inset to Fig. 2 illustrates the challenge in determining a precise value for the boundary of the region of stability. Nevertheless, we can easily place lower bounds on the critical layer separation at a given mass ratio. As can be seen in Fig. 2 and Table 1 . trions are stable over a large region of the $\left(d / a_{0}^{*}, \sigma\right)$ model parameter space. When compared with the biexciton stability region, $\frac{17}{17}$ we find that the trion is bound for layer separations over an order of magnitude larger than those for which the biexciton is bound. Trion formation is always 
possible whenever biexcitons are bound, and trion binding energies are typically far larger for the same set of material parameters.

TABLE I. Critical layer separations for negative trions $\left(d_{\mathrm{X}^{-}}^{\text {crit }}\right)$ and biexcitons $\left(d_{\mathrm{XX}}^{\text {crit }}\right)$ at different electron-hole mass ratios $\sigma$. The biexciton critical layer separations were evaluated using Eq. (2) of Ref. 17.

\begin{tabular}{lcc}
\hline \hline$\sigma$ & $d_{\mathrm{X}}^{\text {crit }} / a_{0}^{*}$ & $d_{\mathrm{XX}}^{\text {crit }} / a_{0}^{*}($ Ref. $[17)$ \\
\hline $1 / 4$ & $4.52(4)$ & 0.48 \\
$1 / 2$ & $5.26(6)$ & 0.43 \\
$3 / 4$ & $6.63(9)$ & 0.42 \\
1 & $7.69(7)$ & 0.42 \\
$4 / 3$ & $>8$ & 0.42 \\
2 & $>8$ & 0.43 \\
4 & $>8$ & 0.48 \\
\hline \hline
\end{tabular}

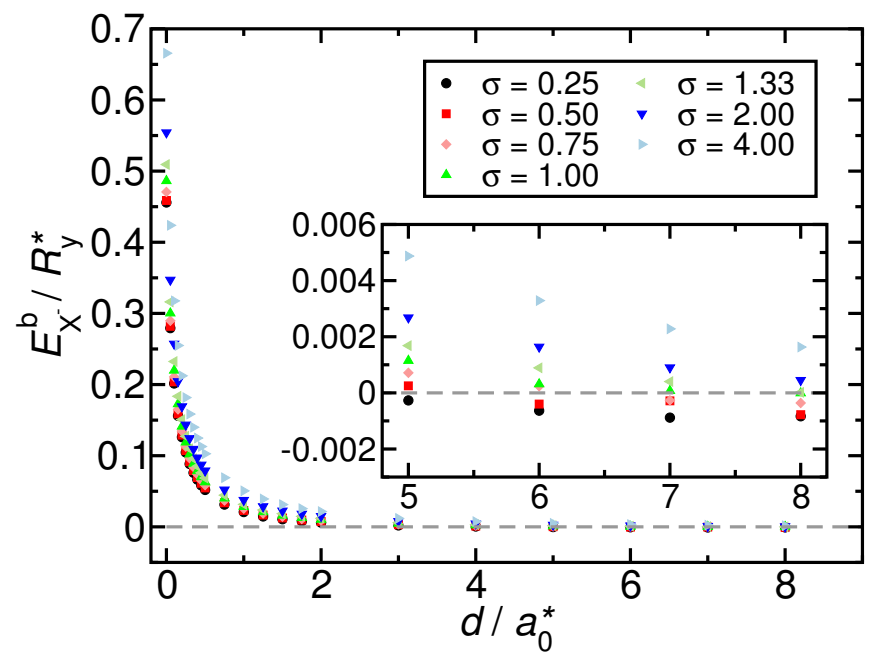

FIG. 2. (Color online) Negative-trion binding energy as a function of interlayer spacing $d$ and electron-hole mass ratio $\sigma$, in excitonic units. The inset shows the edge of the region of stability for negative trions in greater detail.

The biexciton $(\mathrm{XX})$, whose dominant decay is into a pair of excitons $(\mathrm{XX} \rightarrow \mathrm{X}+\mathrm{X}$ ), has a stability region that is determined by the effective interactions of the constituent indirect excitons, and this effective interaction is a repulsive dipole-dipole interaction at long range. $\frac{15 \mid 17}{}$ Negative-trion dissociation is determined by the effective interaction of a lone electron with a single indirect exciton. The interaction potential between an indirect exciton and a lone electron consists of a repulsive part due to the static charge distribution of the exciton, which falls off as $r^{-3}$, and an attractive part due to the induced dipole moment of the exciton, which falls off as $r^{-4}$. Over the intermediate range the attractive part of the interaction plays a much more important role in the trion than in the biexciton.

Fitting Eq. 10 to our trion binding energies results in a maximum error of $5 \times 10^{-4} R_{\mathrm{y}}^{*}$, with over $90 \%$ of the data points falling within $2 \times 10^{-4} R_{\mathrm{y}}^{*}$ of the fit. The fitting parameters $f_{i j}$ and $g_{k}$ are 43

$$
\begin{aligned}
f & =\left(\begin{array}{cccc}
1.408 & 21.53 & 25.25 & 1.676 \\
-2.340 & -40.43 & -36.22 & -11.51 \\
1.617 & 30.47 & 5.803 & 17.36 \\
-0.2129 & -0.5492 & 7.423 & -8.694
\end{array}\right) \\
\mathbf{g} & =\left(\begin{array}{l}
26.16 \\
147.7 \\
186.4 \\
29.45
\end{array}\right)
\end{aligned}
$$

This fit applies only for $1 / 4 \leq \sigma \leq 4$ and $0 \leq d \leq$ $\min \left\{8 a_{0}^{*}, d_{\mathrm{X}^{-}}^{\text {crit }}\right\}$. Accurately parameterizing the binding energy near the critical separation $d_{\mathrm{X}^{-}}^{\text {crit }}$ is not possible with our limited data, and caution should be applied when relying on this fit near the critical region.

Appropriate model parameters for the GaAs/AlGaAs coupled quantum-well device studied by Butov et al $l^{6}$ were identified in Ref. 33. In particular the layer separation $d$ was chosen such that the exciton binding energy obtained using the screened Coulomb interaction with strictly $2 \mathrm{D}$ electrons and holes matches the exciton binding energy obtained using a more realistic model for electrons and holes moving in the quantum wells ${ }^{44}$ The electron and hole masses are taken to be $m_{\mathrm{e}}=0.067 m_{0}$ and $m_{\mathrm{h}}=0.134 m_{0}$, where $m_{0}$ is the bare electron mass. The permittivity is taken to be $\epsilon=13.2 \epsilon_{0}$. Hence the mass ratio is $\sigma=0.5$, the exciton Bohr radius is $a_{0}^{*}=156 \AA$, and the exciton Rydberg is $R_{\mathrm{y}}^{*}=3.5 \mathrm{meV}$. Finally, the layer separation is taken to be $d=100 \AA=0.64 a_{0}^{*}$.

With these parameters, Lee et al ${ }^{17}$ found the critical layer separation for biexciton formation to be $d_{\mathrm{XX}}^{\text {crit }}(0.5)=$ $0.43(5) a_{0}^{*}=67(8) \AA$. This is significantly less than the actual layer separation, implying that biexcitons are unbound. On the other hand, we find the critical layer separation for negative-trion formation to be $d_{\mathrm{X}^{-}}^{\text {crit }}(0.5)=$ $5.26(6) a_{0}^{*}=821(9) \AA$ and the critical layer separation for positive-trion formation to be $d_{\mathrm{X}^{-}}^{\text {crit }}(2)>8 a_{0}^{*}=1248$ $\AA$. Both of these are many times larger than the actual layer separation, implying that both positive and negative trions are bound. Using Eq. (9), the predicted binding energies of negative and positive trions are $0.0411(4) R_{\mathrm{y}}^{*}=0.14 \mathrm{meV}$ and $0.06166(3) R_{\mathrm{y}}^{*}=0.22 \mathrm{meV}$, respectively. Hence both positive and negative trions are expected to be present at temperatures below $T=2 \mathrm{~K}$, which corresponds to an energy of about $k_{\mathrm{B}} T=0.17$ meV.

Our results show that isolated trions are bound in realistic coupled-quantum-well geometries. By continuity, we expect trions to persist as quasiparticles at low, finite charge-carrier concentrations in these systems. A well-defined direct trion persists in a host monolayer $2 \mathrm{D}$ 
electron gas at low or intermediate electron concentrations, up to the point where the density parameter $r_{\mathrm{s}}$ is about three times the exciton Bohr radius ${ }^{41}$ Screening of the electron-hole attraction by a finite concentration of charge carriers in an electron-hole bilayer is expected to reduce the binding energy of an indirect trion and hence to reduce the critical layer separation relative to that of an isolated trion.

Sergeev and Suris have studied indirect trions in coupled quantum wells using the same model as us, but making use of a heavy-hole approximation. ${ }^{31}$ They used a variational approach to find an approximate solution to the 2D Schrödinger equation of a single electron in one layer moving in the potential supplied by two fixed holes in the other layer. The resulting ground-state energy as a function of hole separation provided a BornOppenheimer potential-energy surface for the two holes. They then numerically solved the Schrödinger equation for the relative motion of the holes in that potentialenergy surface to predict $\mathrm{X}^{+}$binding energies in GaAs and $\mathrm{ZnSe}$ coupled quantum wells. In the case of GaAs, at a layer separation of $d=0.42 a_{0}^{*}$, Sergeev and Suris predict an $\mathrm{X}^{+}$binding energy of $0.43 \mathrm{meV}$, whereas the fit to our DMC results (after charge conjugation) yields $0.63 \mathrm{meV}$. In the case of ZnSe, at $d=1.25 a_{0}^{*}$, Sergeev and Suris predict a binding energy of $0.48 \mathrm{meV}$; however fits to our DMC results yield $0.77 \mathrm{meV}$. In order to make these comparisons as fair as possible, we have taken excitonic Rydberg units and electron-hole mass ratios identical to Sergeev and Suris (for GaAs, $R_{\mathrm{y}}^{*}=4.84 \mathrm{meV}$ and $\sigma=0.196$; for $\mathrm{ZnSe}, R_{\mathrm{y}}^{*}=20 \mathrm{meV}$ and $\left.\sigma=0.26\right)$.

\section{B. Biexciton binding energy}

In an extension to the earlier work of Lee et al.,, 17 for completeness we provide an accurate parameterization of the biexciton binding energy in the bound region. We have used the same trial wave function form as in Ref. 33. multiplied by a polynomial Jastrow factor ${ }^{[3435} \mathrm{We}$ have fitted the function

$$
\frac{E_{\mathrm{XX}}^{\mathrm{b}}\left(\sigma, d / a_{0}^{*}\right)}{R_{\mathrm{y}}^{*}}=\frac{\sum_{i=0}^{2} \sum_{j=0}^{2} F_{i j}\left(\sigma+\sigma^{-1}\right)^{i / 2}\left(d / a_{0}^{*}\right)^{j}}{\sum_{k=0}^{3} G_{k}\left(d / a_{0}^{*}\right)^{k}}
$$

to our biexciton binding-energy data, where $F_{i j}$ and $G_{k}$ are fitting parameters. This obeys the necessary symmetry under charge conjugation $\left(\sigma \rightarrow \sigma^{-1}\right)$. As in Eq. 10, the square-root behavior in Eq. 15) arises from harmonic motion in the Born-Oppenheimer approximation.25 Our fit of Eq. 15 has a maximum error of $2 \times 10^{-2} R_{\mathrm{y}}^{*}$, with over $90 \%$ of the data points falling within $5 \times 10^{-3} R_{\mathrm{y}}^{*}$ of the fit. The results of this fit are

$$
\begin{aligned}
F & =\left(\begin{array}{ccc}
0.03495 & -0.9822 & 2.437 \\
0.07670 & 0.3303 & -1.786 \\
-0.005277 & -0.02931 & 0.2942
\end{array}\right) \\
\mathbf{G} & =\left(\begin{array}{c}
0.1726 \\
3.256 \\
1.567 \\
29.95
\end{array}\right) .
\end{aligned}
$$

\section{PDFs}

In Fig. 3 we plot electron-electron and electron-hole pair-distribution functions for negative trions. It is clear that the spatial extent of the trion increases rapidly with layer separation, but is relatively insensitive to mass ratio.
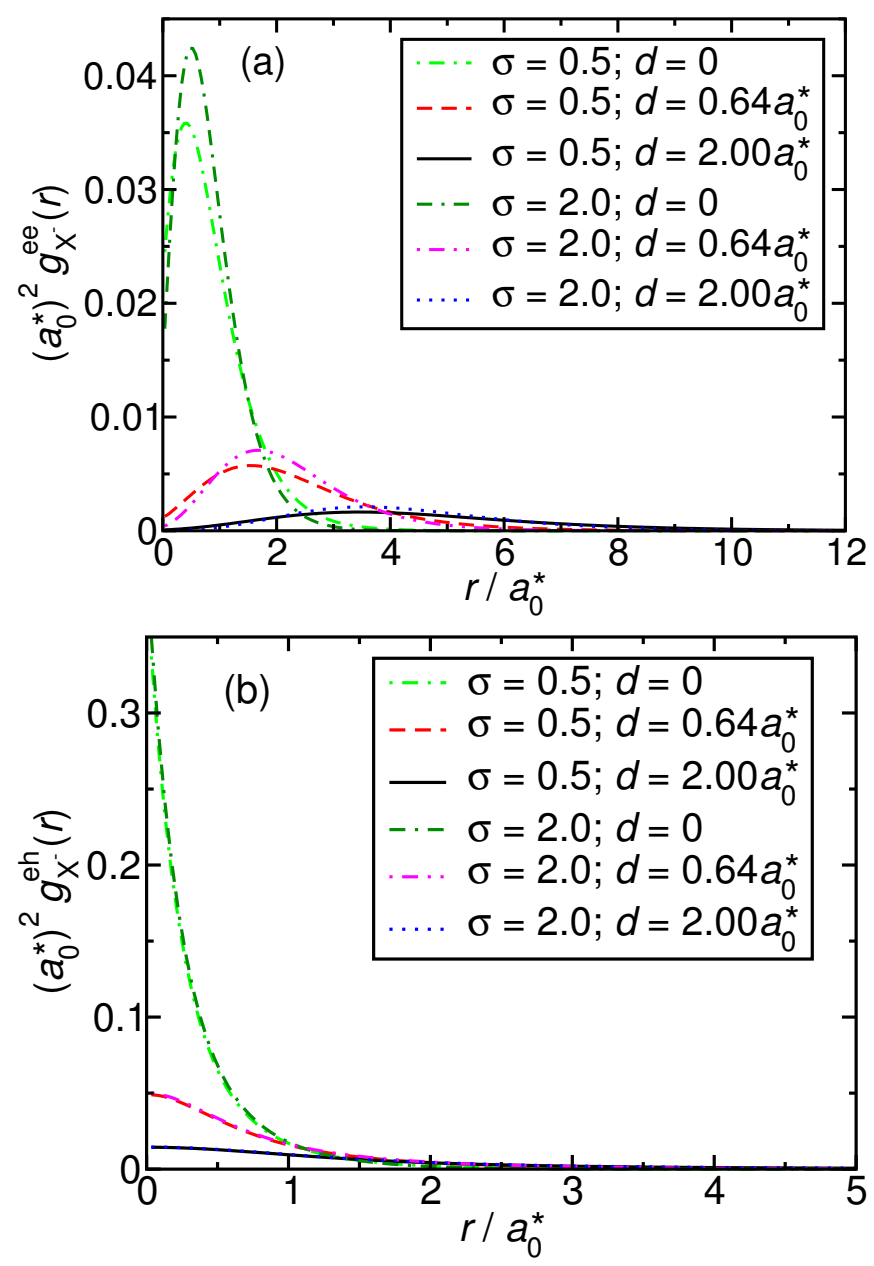

FIG. 3. (Color online) (a) Electron-electron $\left[g_{\mathrm{X}^{-}}^{\text {ee }}(r)\right]$ and (b) electron-hole $\left[g_{\mathrm{X}^{-}}^{\mathrm{eh}}(r)\right]$ pair-distribution functions for indirect negative trions in bilayers with different separations $d$ and electron-hole mass ratios $\sigma$. The $x$-axis shows the in-plane separation. 


\section{CONCLUSIONS}

We have generated statistically exact total-energy data for indirect trions and biexcitons in a simple model of charge-carrier complexes in coupled quantum-well heterostructures. We have found that for indirect trions, the critical layer separation at which the trion becomes unbound is at least an order of magnitude larger than that of the biexciton.

We have applied our results to the coupled quantumwell device studied by Butov et al.,, as modelled by Tan et $a l .{ }^{33}$ We find that, although biexcitons are unbound in this system, positive and negative trions are bound, with substantial binding energies. Qualitatively similar physics is expected in bilayers of $2 \mathrm{D}$ materials, where the interlayer and intralayer charge-charge interaction potentials reduce to the Coulomb $1 / r$ form studied here at long range. In $2 \mathrm{D}$ materials the binding energy of the trion relative to the biexciton is further magnified by the nonlocal screening of the charge carriers by the 2D layers. 23

\section{ACKNOWLEDGMENTS}

Computational resources were provided by Lancaster University's High-End Computing facility. R. J. H. is fully funded by the Graphene NOWNANO centre for doctoral training (grant no. EP/L01548X/1). We acknowledge useful conversations with R. J. Needs and M. Szyniszewski.
1 J. M. Blatt, K. W. Böer, and W. Brandt, Phys. Rev. 126, 1691 (1962)

${ }^{2}$ L. V. Keldysh and A. N. Kozlov, Sov. Phys. J. Exp. Theor. Phys. 27, 521 (1968)

${ }^{3}$ Y. E. Lozovik and V. I. Yudson, J. Exp. Theor. Phys. Lett. 22, 274 (1975)

${ }^{4}$ X. Zhu, P. B. Littlewood, M. S. Hybertsen, and T. M. Rice, Phys. Rev. Lett. 74, 1633 (1995)

5 M. Combescot, O. Betbeder-Matibet, and R. Combescot, Phys. Rev. Lett. 99, 176403 (2007).

6 L. V. Butov, A. C. Gossard, and D. S. Chemla, Nature 418, 751 (2002)

7 D. Snoke, S. Denev, Y. Liu, L. Pfeiffer, and K. West, Nature 418, 754 (2002)

8 S. Yang, A. T. Hammack, M. M. Fogler, L. V. Butov, and A. C. Gossard, Phys. Rev. Lett. 97, 187402 (2006)

${ }^{9}$ V. B. Timofeev and A. V. Gorbunov, Phys. Status Solidi C 5, 2379 (2008)

io A. A. High, J. R. Leonard, A. T. Hammack, M. M. Fogler, L. V. Butov, A. V. Kavokin, K. L. Campman, and A. C. Gossard, Nature 483, 584 (2012)

11 Y. Shilo, K. Cohen, B. Laikhtman, K. West, L. Pfeiffer, and R. Rapaport, Nat. Commun. 4, 2335 (2013)

12 M. Alloing, M. Beian, M. Lewenstein, D. Fuster, Y. González, L. González, R. Combescot, M. Combescot, and F. Dubin, Europhys. Lett. 107, 10012 (2014)

13 M. Combescot, R. Combescot, and F. Dubin, Rep. Prog. Phys. 80, 066501 (2017).

${ }^{14}$ R. Zimmermann and C. Schindler, Solid State Commun. 144, 395 (2007)

is C. Schindler and R. Zimmermann, Phys. Rev. B 78, 045313 (2008)

${ }^{10}$ A. D. Meyertholen and M. M. Fogler, Phys. Rev. B 78, 235307 (2008)

${ }^{17}$ R. M. Lee, N. D. Drummond, and R. J. Needs, Phys. Rev. B 79, 125308 (2009)

${ }^{18}$ K. F. Mak, K. He, C. Lee, G. H. Lee, J. Hone, T. F. Heinz, and J. Shan, Nat. Mater. 12, 207 (2013)

19 C. Zhang, H. Wang, W. Chan, C. Manolatou, and F. Rana, Phys. Rev. B 89, 205436 (2014).

${ }^{20}$ A. Srivastava, M. Sidler, A. V. Allain, D. S. Lembke, A. Kis, and A. Imamoglu, Nat. Phys. 11, 141 (2015).
${ }^{21}$ D. K. Zhang, D. W. Kidd, and K. Varga, Nano Letters 15, $7002(2015)$

${ }^{22}$ A. M. Jones, H. Yu, N. J. Ghimire, S. Wu, G. Aivazian, J. S. Ross, B. Zhao, J. Yan, D. G. Mandrus, D. Xiao, W. Yao, and X. Xu, Nat. Nanotechnol. 8, 634 (2013).

23 M. Szyniszewski, E. Mostaani, N. D. Drummond, and V. I. Fal'ko, Phys. Rev. B 95, 081301 (2017)

24 L. V. Keldysh, J. Exp. Theor. Phys. 29, 658 (1979).

25 E. Mostaani, M. Szyniszewski, C. H. Price, R. Maezono, M. Danovich, R. J. Hunt, N. D. Drummond, and V. I. Fal'ko, Phys. Rev. B 96, 075431 (2017)

26 D. M. Ceperley and B. J. Alder, Phys. Rev. Lett. 45, 566 (1980)

2 W. M. C. Foulkes, L. Mitas, R. J. Needs, and G. Rajagopal, Rev. Mod. Phys. 73, 33 (2001).

28 D. V. Kulakovskii and Y. E. Lozovik, J. Exp. Theor. Phys. Lett. 76, 516 (2002)

${ }^{29}$ O. L. Berman, R. Y. Kezerashvili, and S. M. Tsiklauri, Int. J. Mod. Phys. B 28, 1450064 (2014)

30 V. M. Kovalev and A. V. Chaplik, J. Exp. Theor. Phys. Lett. 88, 454 (2008).

${ }^{31}$ R. A. Sergeev and R. A. Suris, Semiconductors 37, 1205 (2003)

${ }^{32}$ R. J. Needs, M. D. Towler, N. D. Drummond, and P. López Ríos, J. Phys. Condens. Matter 22, 023201 (2010)

33 M. Y. J. Tan, N. D. Drummond, and R. J. Needs, Phys. Rev. B 71, 033303 (2005).

${ }^{34}$ N. D. Drummond, M. D. Towler, and R. J. Needs, Phys. Rev. B 70, 235119 (2004).

35 P. López Ríos, P. Seth, N. D. Drummond, and R. J. Needs, Phys. Rev. E 86, 036703 (2012).

36 T. Kato, Commun. Pure Appl. Math. 10, 151 (1957)

37 R. T. Pack and W. Byers Brown, J. Chem. Phys. 45, 556 (1966)

38 C. J. Umrigar, K. G. Wilson, and J. W. Wilkins, Phys. Rev. Lett. 60, 1719 (1988).

39 N. D. Drummond and R. J. Needs, Phys. Rev. B 72, 085124 (2005).

40 J. Toulouse and C. J. Umrigar, J. Chem. Phys. 126, 084102 (2007).

41 G. G. Spink, P. López Ríos, N. D. Drummond, and R. J. Needs, Phys. Rev. B 94, 041410 (2016) 
42 D. M. Ceperley and M. H. Kalos, in Monte Carlo methods in statistical physics, edited by K. Binder (Springer-Verlag, Heidelberg, 1979) 2nd ed., p. 145.

43 The top-left element of the matrix $f$ in Eq. 13 is the fitting parameter $f_{00}$, and so on.

44 M. H. Szymanska and P. B. Littlewood, Phys. Rev. B 67, 193305 (2003). 